Journal of Computer Science 6 (2): 224-228, 2010

ISSN 1549-3636

(C) 2010 Science Publications

\title{
Iterative Channel Estimation Algorithm in Multiple Input Multiple Output Orthogonal Frequency Division Multiplexing Systems
}

\author{
${ }^{1}$ Mona Nasseri and ${ }^{2}$ Hamidreza Bakhshi \\ ${ }^{1}$ Department of Electrical, Science and Research Branch, Islamic Azad University, Tehran, Iran \\ ${ }^{2}$ Department of Electrical, Shahed University Tehran, Iran
}

\begin{abstract}
Problem statement: The objective of this study is improving channel estimation accuracy in MIMO-OFDM system because channel state information is required for signal detection at receiver and its accuracy affects the overall performance of system and it is essential to improve the channel estimation for more reliable communications. MIMO-OFDM system was chosen in this study because it has been widely used today due to its high data rate, channel capacity and its adequate performance in frequency selective fading channels. For this purpose a $2 \times 2$ system was designed and pilot aided channel estimation with interpolation, is made iterative to enhance BER performance. Approach: First of all, pilots were inserted among subcarriers in transmitter with distances emerged of sampling theory then Least Square (LS) method was chosen for initial channel estimation in pilots at receiver, using applicable proposed receiver, which has simple and usable structure, then channel state information was estimated by linear interpolator in information subcarriers, which uses two adjacent channel estimation in pilots to compute channel in another subcarriers and an LMS iterative algorithm, including a feedback of output is added to system. This algorithm uses the channel estimation of last iteration in current estimation. Results: Adding a LMS iterative algorithm to system, improves the channel estimation performance. Simulation results proved the acceptable BER performance of iterative channel estimation algorithm, which is closed to the ideal channel. Conclusion: The lowcomplexity proposed receiver including LMS algorithm, has a higher efficiency than conventional methods and it can work in lower amount of SNRs.
\end{abstract}

Key words: MIMO-OFDM, pilot aided channel estimation, linear interpolator, LMS iterative algorithm

\section{INTRODUCTION}

OFDM is a modulation technique, which is used in frequency selective fading channels, divides a high data rate into a number of lower data rate streams over subcarriers transmitted simultaneously through channel. Each sub stream experiences flat fading narrowband channel (Proakis, 2001).

Subcarriers are orthogonal in time domain, which save bandwidth in frequency domain, resulted in ICI cancelation. Additionally, CP (Nee and Prasad, 2000) as a guard interval with a length, longer than delay spread, that is a copy of last $\mathrm{N}_{\mathrm{g}}$ samples, in front of each symbol, avoids ISI (Nee and Prasad, 2000), therefore OFDM technique is a method to remove the effects of frequency selective fading channels and to enhance the bandwidth efficiency up to 50\% comparing with FDM technique.
OFDM system is combined with multiple antenna in both sides of link, resulted in MIMO-OFDM system and higher channel capacity (Telatar, 1999).

Channel information is required at receiver for signal detection. However, There are different methods of channel estimation such as pilot aided ( $\mathrm{Li}, 2002)$ and blind (Gao and Nallanathan, 2007) approaches, the first method is chosen as a channel estimation method in this study due to its less complexity. According to sampling theory (Oppenheim and Schafer, 1999), Pilots are inserted equal-spaced among subcarriers in frequency domain at transmitter, which are known at receiver and will be extracted to estimate channel at pilot subcarriers and interpolation is implemented for channel estimation in another subcarriers. In the analysis, channel is estimated with LS (Coleri et al., 2002) method at pilots, then linear interpolation is used to complete the estimation (Coleri et al., 2002; Hsieh and Wei, 1998).

Corresponding Author: Mona Nasseri, Department of Electrical, Science and Research Branch, Islamic Azad University, Tehran, Iran Tel: +989121468603 
For improving the quality of channel estimation, different iterative algorithms are available, such as EM (Xie and Georghiades, 2003), RLS and LMS (Haykin, 1996), among them LMS is chosen due to its less complexity comparing with other methods and its acceptable performance.

In this study the system model are introduced in section which followed by implementing channel estimation by proposed receiver which are improved by iterative algorithm and observing the simulation result, followed by a discussion.

\section{MATERIALS AND METHODS}

The general system model is introduced then it is described in detail with a complete explanation of receiver and the iterative algorithm, which was used.

System model: a $2 \times 2$ MIMO-OFDM system is considered, which is shown in Fig. 1.

A symbol of length $\mathrm{N}$ is considered, which $\mathrm{P}$ pilots are inserted among data in distances, emerged of sampling theory (Oppenheim and Schafer, 1999), to avoid aliasing, as follows:

$$
\mathrm{N}_{\mathrm{f}}<\frac{\mathrm{N}}{\mathrm{N}_{\mathrm{g}}}, \quad \mathrm{N}_{\mathrm{t}}<\frac{1}{2\left(1+\frac{\mathrm{N}_{\mathrm{g}}}{\mathrm{N}}\right) \mathrm{f}_{\mathrm{D}, \max }}
$$

Assuming the pilots are placed $\mathrm{N}_{\mathrm{f}}$ subcarriers apart in every $\mathrm{N}_{\mathrm{t}}$ OFDM symbols and $\mathrm{f}_{\mathrm{D} \text {, max }}$ is the maximum Doppler frequency. The pilots are chosen randomly by BPSK modulation and data has QPSK modulation due to its higher bandwidth efficiency (Xiong, 2000). The modeling of the system is as follows:

$$
\mathrm{Y}=\mathrm{HX}+\mathrm{W}
$$

Where:

$\mathrm{Y}=$ Received signal matrix in frequency domain

$\mathrm{X}=$ Transmitted signal

$\mathrm{W}=\mathrm{AWGN}$

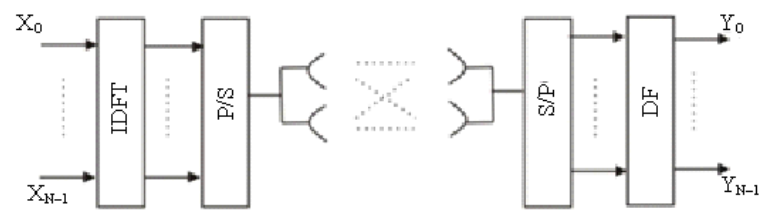

Fig. 1: The schematic structure of MIMO-OFDM system
Also $\mathrm{H}$ channel matrix can be shown as:

$\mathrm{H}=\left[\begin{array}{ccc}\mathrm{H}_{1,1} & \cdots & \mathrm{H}_{1, \mathrm{n}_{\mathrm{t}}} \\ \vdots & \ddots & \vdots \\ \mathrm{H}_{\mathrm{n}_{\mathrm{r}}, 1} & \cdots & \mathrm{H}_{\mathrm{n}_{\mathrm{r}} \times \mathrm{n}_{\mathrm{t}}}\end{array}\right]$

where the first subscript shows the receive antenna and the second one is transmit antenna. $\mathrm{H}_{\mathrm{i}, \mathrm{j}}=\operatorname{diag}\left[\mathrm{H}_{0} \ldots\right.$ $\left.\mathrm{H}_{\mathrm{N}-1}\right]$, defines the channel between jth transmit antenna and ith receive antenna.

Receiver designing: At the receiver, $n_{r} \times n_{t}$ sets of extracted received pilot tones are used for channel estimation, which LS method is chosen due to its simplicity. The standard formula for this approach at mth symbol is computed as:

$$
\mathrm{H}_{\mathrm{LS}}(\mathrm{m})=\left(\left(\mathrm{X}^{\mathrm{P}}(\mathrm{m})\right)^{\mathrm{H}} \mathrm{X}^{\mathrm{P}}(\mathrm{m})\right)^{-1}\left(\mathrm{X}^{\mathrm{P}}(\mathrm{m})\right)^{\mathrm{H}} \mathrm{Y}^{\mathrm{P}}(\mathrm{m})
$$

where, $\mathrm{X}^{\mathrm{P}}(\mathrm{m})$ and $\mathrm{Y}^{\mathrm{P}}(\mathrm{m})$ respectively show the transmitted and received pilots. After channel estimation in pilot tones, channel is estimated in all subcarriers, using linear interpolator:

$\hat{H}\left(1 \times \frac{N}{L}+n\right)=\hat{H}\left(p(1)+n \times \frac{\hat{H}(p(1+1))-\hat{H}(p(l))}{N / L}\right.$
$0 \leq n<\frac{N}{L}, 0 \leq 1<L$

where, $\mathrm{L}$ is the number of pilots and the equation computes the channel in $(1 \times \mathrm{N} / \mathrm{L}+\mathrm{n})$ th subcarrier and $\mathrm{p}(1+1)$ and $\mathrm{p}(\mathrm{l})$ show the pilots location. Two adjacent channel estimation through LS estimation are used to estimate channel in subcarriers between them.

The $n_{r} \times n_{t}$ matrix of channel estimation is applied to the whole received symbol. For each receive antenna, $\mathrm{n}_{\mathrm{t}}$ data stream are added to produce the signal. The final signal at each receiver, after detection is computed as:

$$
\mathrm{R}_{\mathrm{i}}=\operatorname{de} \bmod \left(\frac{1}{\mathrm{n}_{\mathrm{t}}}\left(\sum_{\mathrm{n}=1}^{\mathrm{n}_{\mathrm{t}}} \mathrm{Y}_{\mathrm{n}} / \hat{\mathrm{H}}_{\mathrm{n}}\right)\right)
$$

where channels are considered with equal power, otherwise the coefficient related to the power of channel, should be multiplied in $\mathrm{H}_{\mathrm{i}, \mathrm{j}}$. The structure of proposed receiver is shown in Fig. 2. Which is considered for one receiver, $\mathrm{R}_{\mathrm{XS}} 1$ and $\mathrm{R}_{\mathrm{XS}} 2$ are the data were received through 2 paths. 


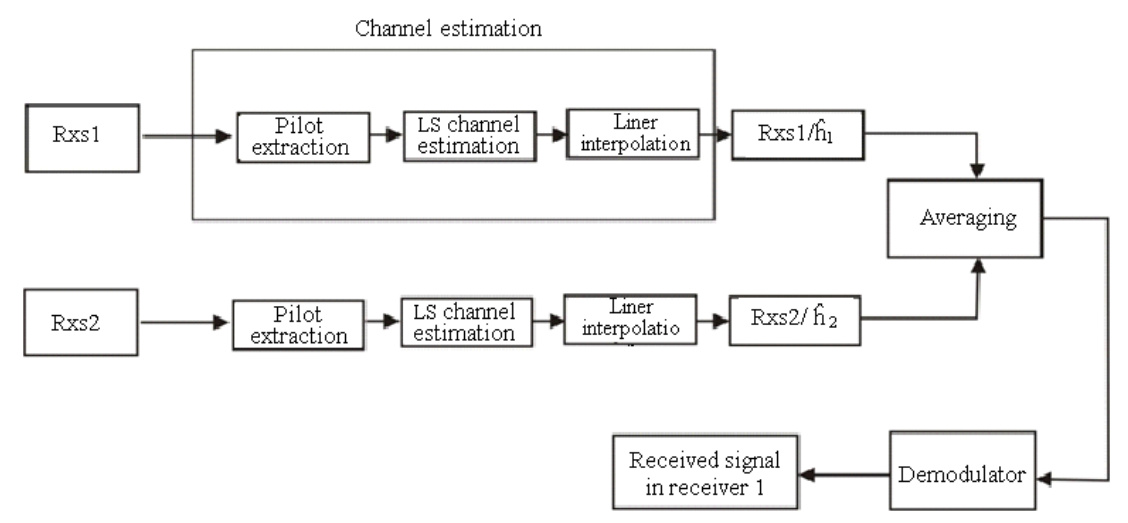

Fig. 2: The structure of proposed receiver

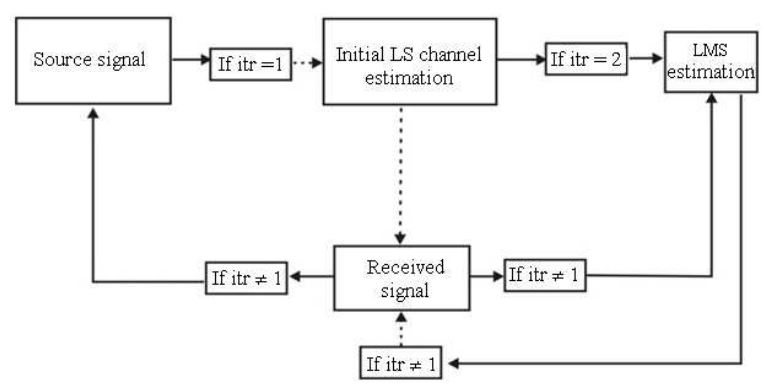

Fig. 3: Implementing LMS algorithm in proposed receiver

Iterative algorithm: There are different iterative algorithms which are used to improve channel estimation and various methods are obtained as initial estimation. Also estimation in each iteration, can be used as side information and feed back to system to achieve better result in next iteration. Some of this methods are used for SISO and MIMO systems (Chen et al., 2007; Qiao et al., 2005). In this study for initial channel estimation, data aided schemes are used and pilots are transmitted along with data. The iterative algorithm which is used in this article, is LMS algorithm, that uses channel estimation of last iteration. It is given by:

$$
\hat{\mathrm{H}}_{\mathrm{n}}=\hat{\mathrm{H}}_{\mathrm{n}-1}-\mu \times \mathrm{e} \times \mathrm{X} *
$$

Where subscript:

$\mathrm{n}=$ The iteration state

$\mathrm{e}=$ The signal error

$\mu=$ A coefficient between $0-1$

The iteration process is continued until the determined criteria will be reached, which it could be an especial MSE.
As illustrated in Fig. 3, LMS algorithm is applied to receiver and the channel which was estimated in each iteration would be used for next iteration, additionally the output signal is fed to source signal for next channel estimation. Another important factor in channel estimation through this method is $\mu$ which influences on estimation and should be precisely chosen.

\section{RESULTS}

The $2 \times 2$ MIMO-OFDM system was simulated using matlab program so that pilots are inserted among data for initial LS channel estimation. The channel between transmitter and receiver antennas was modeled by multipath rayleighy fading channel using jakes spectrum type. Channel bandwidth is $1.75 \mathrm{Mhz}$ (IEEE $802.162004,2004)$. The channel simulation parameters such as delay spread, tap power and Doppler frequency were chosen, using Standard University Interim channel model (SUI) (Erceg et al., 2001). This model can be used for simulation because it is suitable for fixed broadband wireless application.

A FFT with the size of 1024 was used, with a CP length of 256. In which data are in QPSK modulation and BPSK modulation was chosen for pilots. The pilots are placed in distance which fulfill the sampling theory, was mentioned in Eq. 1.

At receiver, all of procedures such as adding $\mathrm{CP}$, are reversely done and pilots are extracted after FFT operation for LS estimation and linear interpolation. Fig. 4 shows the performance of proposed receiver, introduced in Fig. 2, for 2 receive antennas and the performance is different for 2 receivers due to the differences of links parameters such as delay and power. 


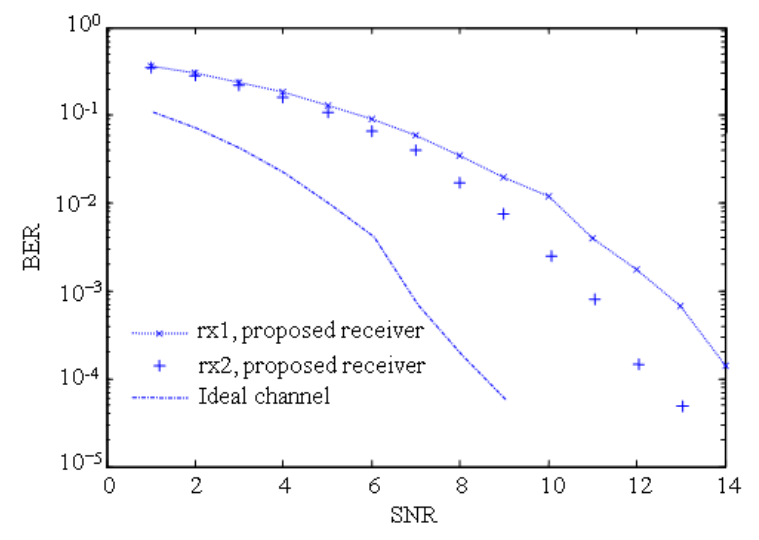

Fig. 4: Performance of proposed receiver

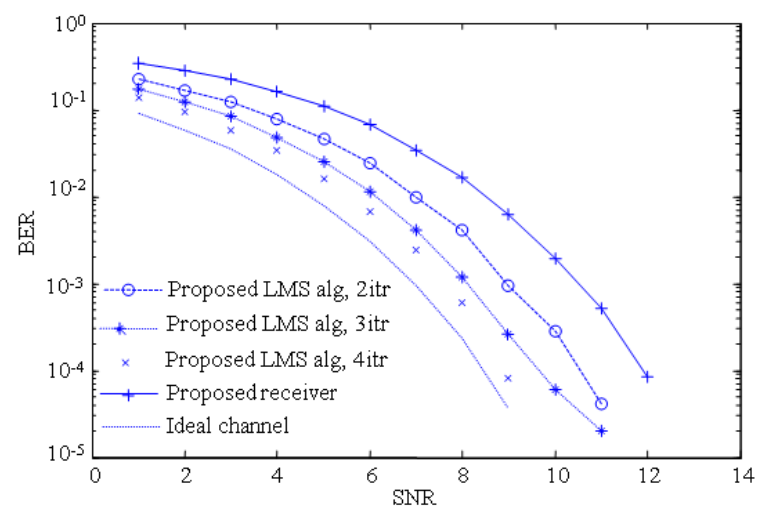

Fig. 5: Improved channel estimation, using LMS iterative algorithm

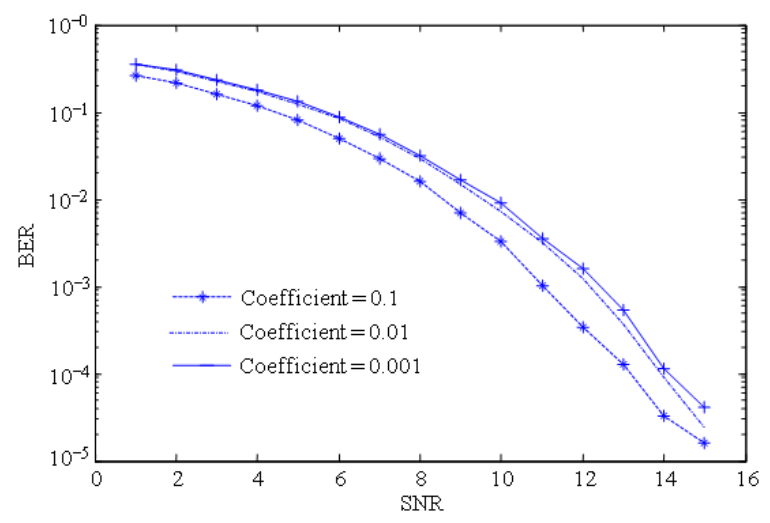

Fig. 6: Effect of $\mu$ changes

Figure 5, illustrates, the estimation can be improved by adding an iterative algorithm at receiver as is demonstrated in Fig. 3, which was shown for one receiver that has better performance than other one. The performance of channel estimation will be better in higher iterations but the changes decreases comparing with less numbers of iteration and a criteria such as MSE can determine the number of iterations. Another factor which influences on performance of system is the value of $\mu$, which can be chosen by experiment and in Fig. 6, its effect was investigated. 0.1 due to its better performance, was used in this study for simulation.

\section{DISCUSSION}

In this study MIMO-OFDM system was chosen for investigation due to its advantages, such as removing ISI and ICI and its appropriate performance in frequency selective fading channels and a simple structure of the receiver for channel estimation was proposed and comparing with Coleri et al. (2002) and Hsieh and Wei (1998), it was designed for MIMO system which has higher efficiency than SISO system also it works in a lower amount of SNR which refer to less energy consumption. In proposed receiver, The LS method was used for initial channel estimation and linear interpolation was chosen for completing the process. The estimation was improved, using LMS iterative algorithm, including a feedback of output to source which lead to lessen error and is simpler than some iterative algorithms such as EM. Its BER performance shows acceptable performance, closed to the ideal channel. The number of iterations can be determined by a criteria such as MSE. Additionally the $\mu$ should be chosen precisely and it can be a good option to research and to use the ways of updating it in each iteration.

\section{CONCLUSION}

In this study a low complexity structure of receiver was proposed so that the LS method and linear interpolation were used for initial channel estimation. For improving accuracy of channel estimation, LMS iterative algorithm was added to receiver which includes a feedback of output and improves the BER performance of system, closed to the ideal channel performance.

For future research, the method to determine the pilots location, to improve efficiency of the system may be considered.

\section{REFERENCES}

Coleri, S., M. Ergen, A. Puri and A. Bahai, 2002. Channel estimation techniques based on pilot arrangement in OFDM systems. IEEE Trans. Broadcast., 48: 223-229. DOI: 10.1109/TBC.2002.804034 
Erceg, V., K.V.S. Hari, M.S. Smith and D.S. Baum, 2001. Channel Models for Fixed Wireless Applications. IEEE 802.16.3 Task Group Contributions.

Chen, J., C. Chiang and L. Lo, 2007. Iterative Channel Estimation for MIMO OFDM Systems. Proceeding of the International Symposium on Intelligent Signal Processing and Communication Systems, Nov. 28-Dec. 1, IEEE Xplore Press, Xiamen, pp: 774-777. 10.1109/ISPACS.2007.4446002

Gao, F. and A. Nallanathan, 2007. Blind channel estimation for MIMO OFDM systems via nonredundant linear precoding. IEEE Trans. Sig. Process., 55: 784-789. DOI: 10.1109/TSP.2006.885764

Haykin, S., 1996. Adaptive Filter Theory. 3rd Edn., Prentice-Hall, New Jersey, ISBN: 0-13-39-7985-7, pp: 506.

Hsieh, M. and C. Wei, 1998. Channel estimation for OFDM systems based on comb-type pilot arrangement in frequency selective fading channels. IEEE Trans. Consum. Elect., 44: 217-225. DOI: $10.1109 / 30.663750$

IEEE 802.162004, 2004. IEEE standard for local and metropolitan area networks, part 16: Air interface for fixed broadband wireless access systems. http://www.citeulike.org/user/damlegone/article/43 26988

Li, Y., 2002. Simplified channel estimation for OFDM systems with multiple transmit antennas. IEEE Trans. Wireless Commun., 1: 67-75. DOI: 10.1.1.110.6351

Nee, R.V. and R. Prasad, 2000. OFDM for Wireless Multimedia Communications. Artech House, ISBN: 0-89006-530-6, pp: 33-50.
Oppenheim, A. and R.Schafer, 1999. Discrete Time Signal Processing. 2nd Edn., Prentice-Hall, ISBN: 0-13-754920-2, pp: 146.

Proakis, J.G., 2001. Digital Communications. 4rd Edn., Mc Graw-Hill, New York, ISBN: 0-07-118183-0, pp: 709-800.

Qiao, Y., S. Yu, P. Su and L. Zhang, 2005. Research on an iterative algorithm of LS channel estimation in MIMO OFDM systems. IEEE Trans. Broadcast., 51: 149-153. DOI: 10.1109/TBC.2004.842524

Telatar, E., 1999. Capacity of multi-antenna Gaussian channels. Eur. Trans. Commun., 10: 585-595.

Xie, Y. and C.N. Georghiades, 2003. Two EM-type channel estimation algorithms for OFDM with transmitter diversity. IEEE Trans. Commun., 51: 106-115. DOI: 10.1109/TCOMM.2002.807617

Xiong, F., 2000. Digital Modulation Techniques. Artech House, ISBN: 0890069700, pp: 123-170. 